demand. This includes polarisation, intensity, and frequency of the generated light and the overall shape of the beam Several mechanisms could be used to generate such light profiles upon electron-beam irradiation. The coherence state and the shape of the generated light are inherently linked to the materials properties, such as either an electron
gas in a metal or collective excitons gas in a metal or collective excitons
in a semiconductor that is excited. In particular, when collective electronic excitations such as plasmon polaritons are used, the spontaneous coherence and sensitivity of the plasmon frequency and their durations to the geometric shape of their host medium allow the shape, frequency and polarisation of the generated light to be designed.

\section{Results to date}

To design the EDPHS, various concepts based on metamaterials, holography, and photon sieves could be employed. We have recently used the latter technique to design and fabricate a photon sieve EDPHS structure that allows for (T) sample (Talebi et al., 2019) (Figure. 2a) or generating light with orbital angular (Figure. 2b) These EDPHS a 2020 are fabricated by incorporating an irregular lattice of holes in a gold thin film deposited on a Si3N4 transmission electron microscopy membrane. The rather simple method of fabricating these structures enables a fabulous techniqu to tailor the properties of the generated light source on demand, e.g. by creating various EDPHS structures in a single membrane and switching between them (Figure 2a to d).

The EDPHS structures are inserted inside an electron microscope and positioned at a certain distance with respect to the sample (Figure 3a). A nanopositioner system is used to align the distance with ultimately angstrom resolution, allowing the control of the dynamics at sub-femtosecond temporal resolutions. Our recent proof of concept experiments
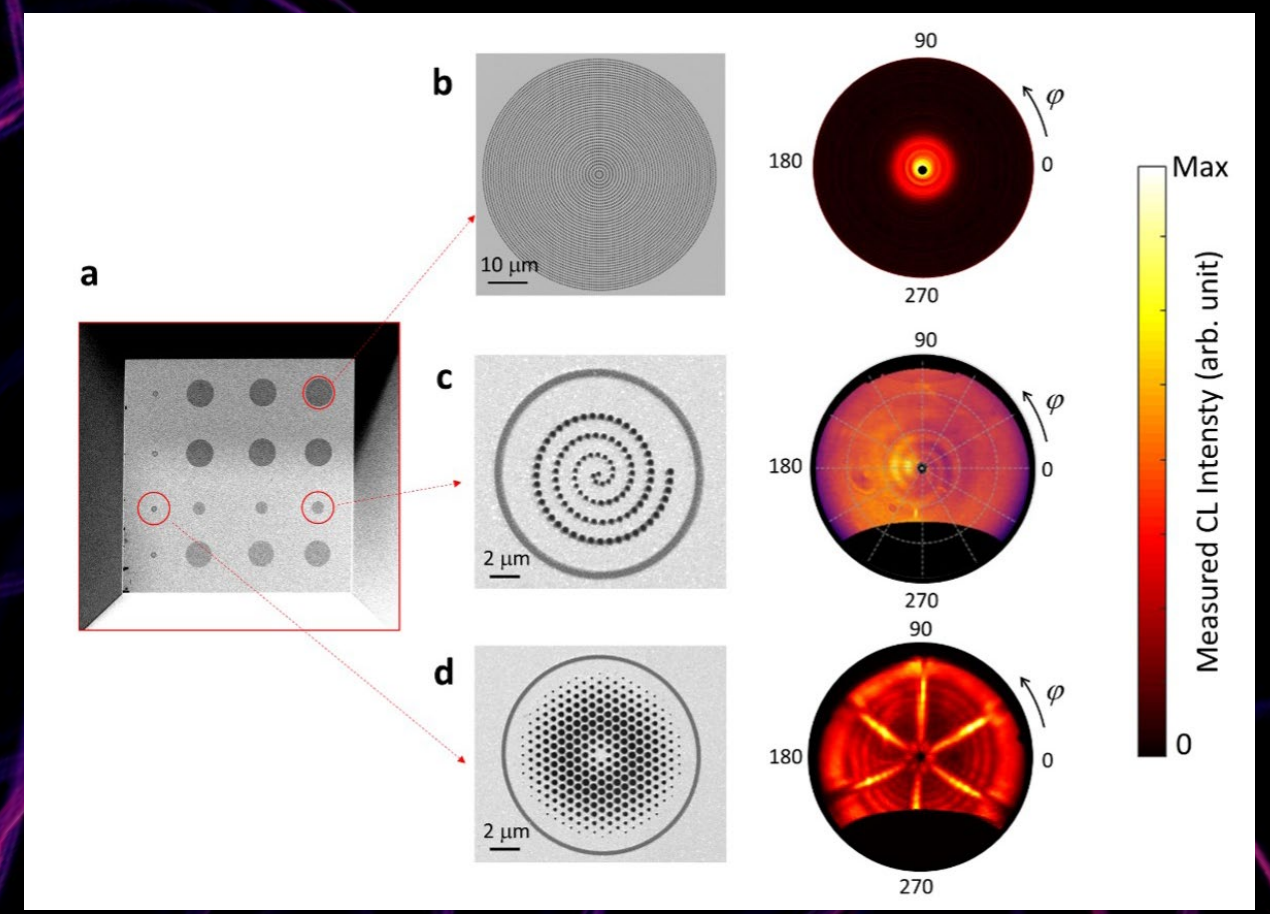

d

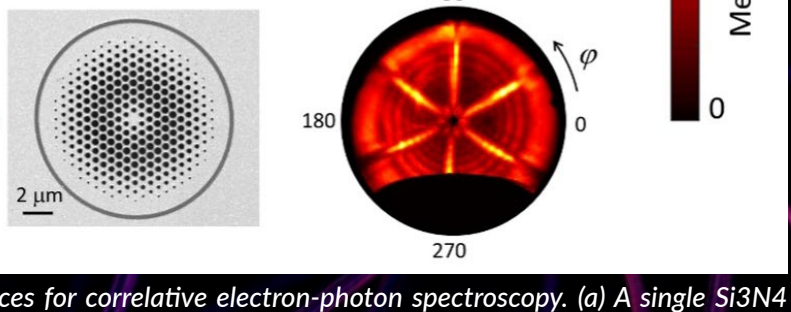

Figure 2: Electron-driven photon sources for correlative electron-photon spectroscopy. (a) A single Si3N4 4
TEM membrane that includes a variety of different photon sive structures that, in interaction with electron beams, generate (b) collimated light, (c) light with angular momentum, or (d) light with a specific symmetric
pattern. Left column in panels (b) to (d) shows the SEM images of the EDPHS structures. The right column
shows the detected angle-resolved pattern of detected photons at the filtered wavelength of $800 \mathrm{~nm}$.

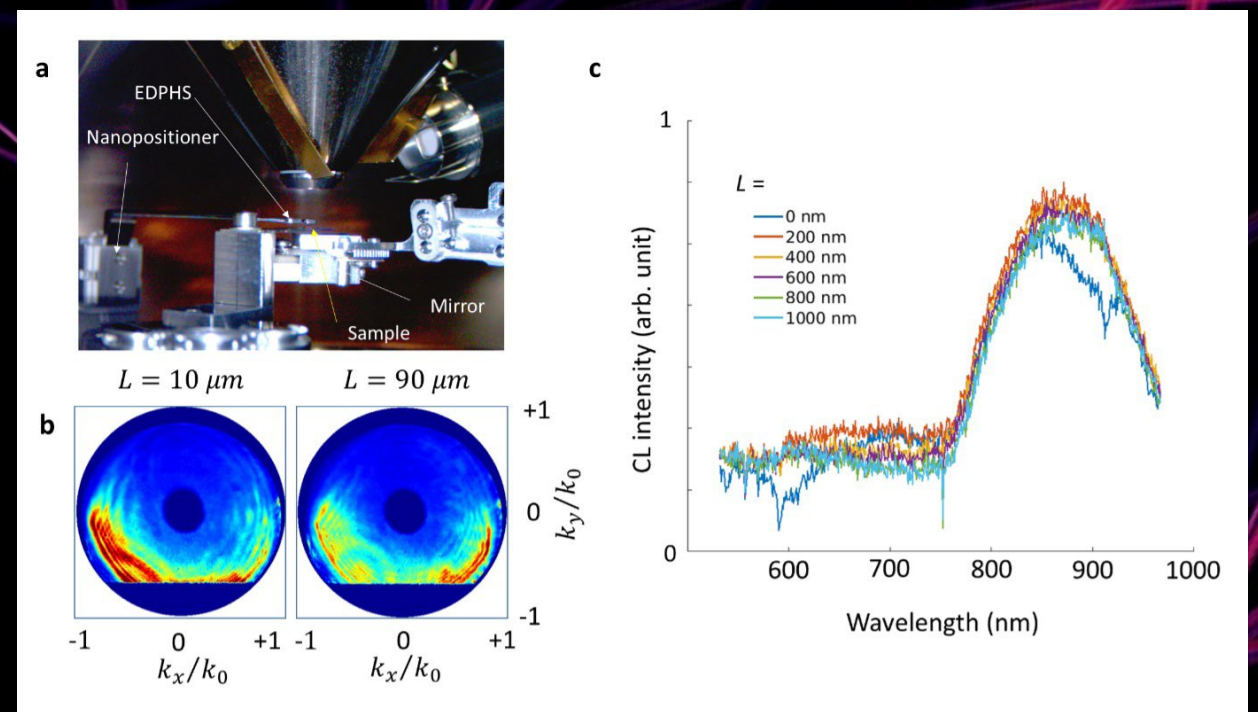

Figure 3: Developed correlative electron-photon spectroscopy technique that utilises an EDPHS Structure.
(a) An image of the setup that contains the nanopositioner and EDPHS, sample stage, and the CEcollector (a) An image of the setup that contains the nanopositioner and EDPHS, sample stage, and the CL collector
(mirror). (b) Acquired angle-resolved pattern at the wavelength of $800 \mathrm{~nm}$ at depicted distances between the (mirror). (b) Acquired angle-resolved pattern at the wavelength of $800 \mathrm{~nm}$ at depicted distances between the
sample and the EDPHS. (c) Acquired spectra at selected distances, where the fluctuations of the spectrum versus distance are apparent.

demonstrate that both the angle-resolved example. The EDPHS structure shown patterns (Figure $3 \mathrm{~b}$ ) and the spectra in Figure $2 \mathrm{~b}$ has been used for obtaining (Figure 3c) demonstrate many interference the results shown in Figure 3. A quantum phenomena that could pave the way material, i.e. a thin flake of a transition towards complementary approaches metal dichalcogenides hosting roomcompared to $4 \mathrm{D}$ electron microscopy temperature exciton polaritons, has been to unravel the photon statistics as an used for the sample. step forward toward our aims for realising Our results perfectly match with the a few-photon spectroscopy technique
milestones of the ERC Starting Grant favouring the electron-light interactions project NanoBeam and are a significant at the extreme level.
Nano

स्ञीज

\section{References}

Christopher, J., Taleb, M., Maity, A., Hentschel, M., Giessen, H. and Talebi, N. (2020) 'Electron-driven photon sources for correlative electron-photon spectro
9(15), 4381 (2020), do: 10.1515/nanoph-2020-0263.

Feist, A., Echternkamp, K. E., Schauss, J., Yalunin, S. V., Schäfer, S. and Ropers, C. (2015) 'Quantum coherent optical phase modulation in an $u$
pp. 200-203. doi: $10.1038 /$ nature14463.

Flannigan, D.J. and Zewail, A.H. (2012) '4D Electron Microscopy: Principles and Applications', Accounts of Flannigan, D.J. and Zewail, A.H. (2012) '4D Electron Microscopy: Princt
Chemical Research, 45(10), pp. 1828-1839. do: 10.1021/ar3001684.

van Nielen, N., Hentschel, M., Schilder, N., Giessen, H., Polman, A. and Talebi, N. (2020) 'Electrons 20, 5975. doi: 10.1021/acs.nanolett.0c01964.

Talebi, N. and Lienau, C. (2019) 'In'th
New Journal of Physics, 21, 093016.

Talebi, N., Meuret, S., Guo, S., Hentschel, M., Polman, A., Giessen, H., and van Aken, P.A. (2019) 'Merging transformation optics with ele $10.1038 / 541467-019-08488-4$.

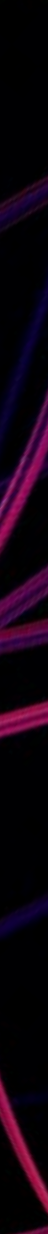
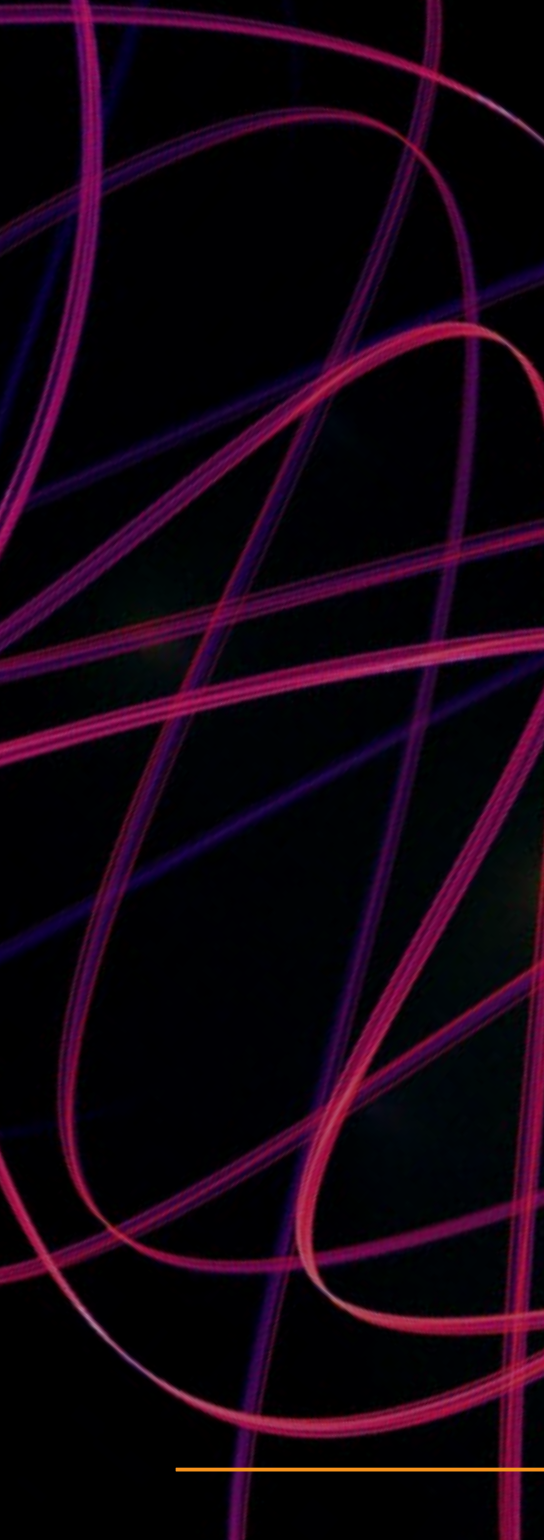

PROJECT SUMMARY

The multidisciplinary ERC Starting Grant project "Quantum Coherent Contro: Self-Interference of Electron Beams with
Nanostructures (NanoBeam)" combines experiments and simulations to develop scanning electron microscopes into
practical tool for probing electron dynamics practical tool for probing electron dynamics
at the nanoscale, opening new vistas for at the nanoscale, opening new vistac

PROJECT LEAD

Born in Iran, Dr Talebi received her awardwinning doctorate in electrical engineering from the University of Terran in 2011 . Shie
joined the Max Planck Institute in 2012 as joined the Max Planck Institute in 2012 and received an ERC starting grant in 2018 to probe electron dynamics using electro 50 peer-review articles and two patents. PROJECT PARTNERS

The NanoBeam project is based at the Max Planck Institute for Solid State Research in Stuttgart, taking advantage of its state-
of -the-art electron microscopy lab and of-the-art electron mutionstional resources. Collaboration parthers include groups from Germa
France, the Netherlands, and bevond.

CONTACT DETAILS

Dr Nahid Talebi Sarvari, PhD
Scientist and Group Leader, Max Planck institute for Solid State Research

Iiiv $+49(0) 7116893649$

n.talebi@fikfmpg.de

(1) www.fikf.mpg.de/6519164/erc-groultalebi

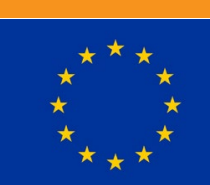

FUNDING This project has received funding from the European
Union's Horizon 2020 research and innovation
nrogeramme under grant agreament No 831851 